\title{
Expression of Sonic Hedgehog and pathway components in the embryonic mouse head: anatomical relationships between regulators of positive and negative feedback
}

\author{
Crystal L. Sigulinsky ${ }^{1}$, Xiaodong $\mathrm{Li}^{2}$ and Edward M. Levine ${ }^{1,2,3^{*}}$ (D)
}

\begin{abstract}
Objective: The Hedgehog pathway is a fundamental signaling pathway in organogenesis. The expression patterns of the ligand Sonic Hedgehog (Shh) and key pathway components have been studied in many tissues but direct spatial comparisons across tissues with different cell compositions and structural organization are not common and could reveal tissue-specific differences in pathway dynamics.

Results: We directly compared the expression characteristics of Shh, and four genes with functional roles in signaling and whose expression levels serve as readouts of pathway activity in multiple tissues of the embryonic mouse head at embryonic day 15.5 by serial in situ hybridization. The four readout genes were the positive feedback regulator Gli1, and three negative feedback regulators, Patched1, Patched2, and Hedgehog Interacting Protein. While the relative abundance of Gli1 was similar across tissues, the relative expression levels and spatial distribution of Shh and the negative feedback regulators differed, suggesting that feedback regulation of hedgehog signaling is context dependent. This comparative analysis offers insight into how consistent pathway activity could be achieved in tissues with different morphologies and characteristics of ligand expression.
\end{abstract}

Keywords: Sonic hedgehog, Gli1, Patched, Hedgehog interacting protein, Gene expression, Retina, Palatal rugae, Hair follicle, Eyelid, Molar, Embryonic day 15.5

\section{Introduction}

Shh is a secreted glycoprotein belonging to the Hedgehog (Hh) family of intercellular signaling molecules. The mechanics of Hh signaling is complex, extending from ligand production through signal transduction to the cell- and tissue-specific responses (reviewed in [14]). In its simplest iteration (Fig. 1A), binding of Shh to its receptor, Patched 1 (Ptch1) or, in some cases, Ptch2,

\footnotetext{
*Correspondence: ed.levine@vumc.org

${ }^{2}$ Department of Ophthalmology and Visual Sciences, Vanderbilt Eye Institute, Vanderbilt University Medical Center, 1161 21st Ave S, B3307 MCN/2569, Nashville, TN 37232, USA

Full list of author information is available at the end of the article
}

relieves inhibition of the G-protein coupled receptor Smoothened (Smo). Activated Smo inhibits proteolytic processing of the GLI transcriptional effectors Gli2 or Gli3 into truncated repressor forms through destabilization of complexes between Gli2 or Gli3 and Suppressor of Fused (Sufu). The resulting accumulation of full-length GLI proteins in the nucleus promotes the expression of Hh target genes.

Transcriptional targets of the pathway not only mediate cellular responses to Hh ligands, but also participate in feedback loops that further regulate pathway activity. The principal positive feedback loop involves the transcriptional effector Gli1. Gli1 expression is activated in response to Gli2 transcriptional activation [5-7]. 

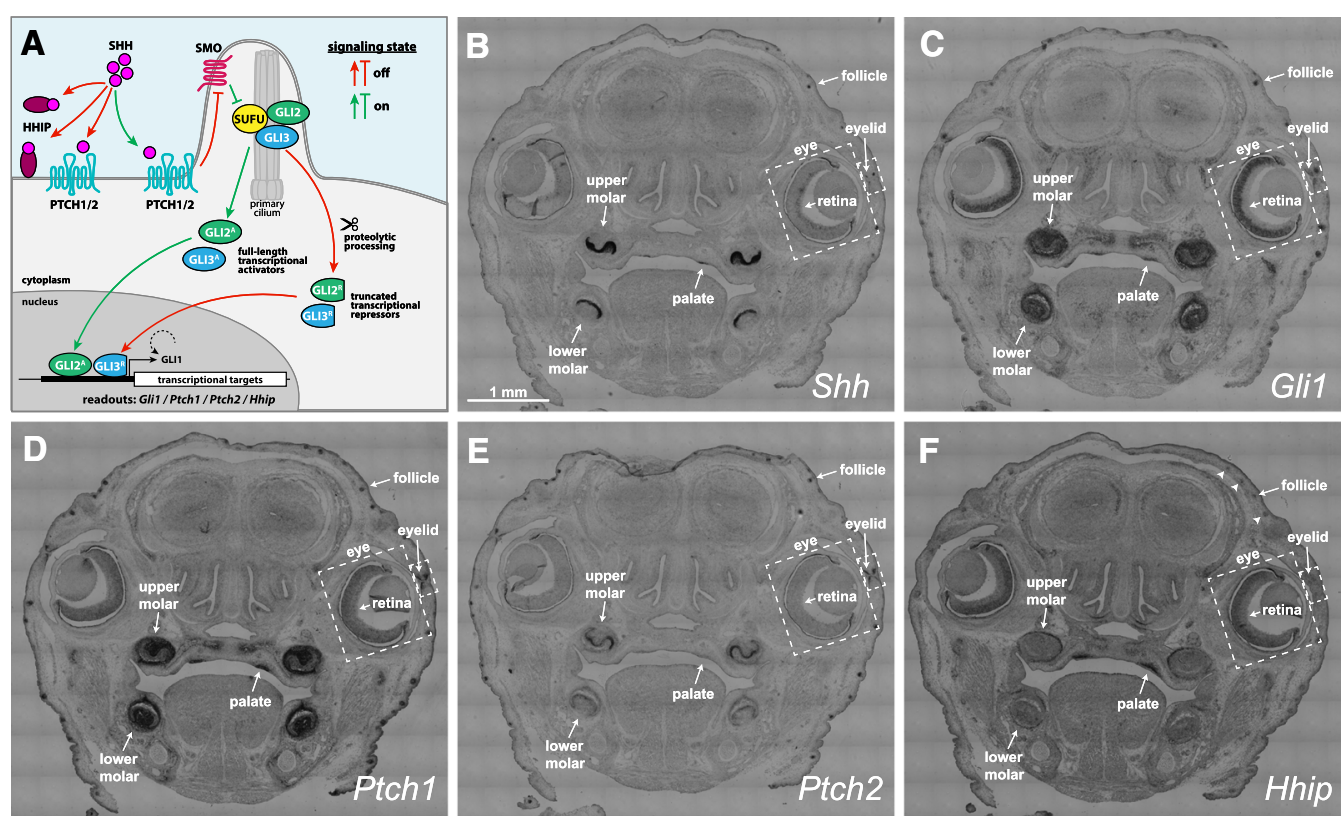

Fig. 1 Expression patterns for Shh and Hh pathway components in developing organs of the embryonic mouse head. A Simplified schematic of Hh signaling. See "Introduction" section for details. B-F in situ hybridizations for Shh (B), Gli1 (C), Ptch1 (D), Ptch2 (E), and Hhip (F) in adjacent coronal sections of the mouse at E15.5. Arrowheads in F denote additional hair follicles

This, together with its activator function, allows Gli1 to increase signaling levels while retaining dependence on active Hh signaling. Gli1 expression is therefore an excellent indicator of pathway activity.

Ptch1, Ptch2, and Hhip participate in negative feedback that act at the level of Hh reception [8-10]. Ptch1 transcripts are also upregulated in response to Hh signaling [11-13], and evaluation of phenotypes and $\mathrm{Hh}$ pathway activity in Ptch1 mutant mice shows that Hh activity is sensitive to Ptch1 gene dosage [14-16]. In addition to Smo inhibition, upregulation of Ptch1 (Ptc in Drosophila) also sequesters Hh ligands and desensitizes the cell to Hh signal [17]. Patch2 shares sequence homology with Ptch1, binds Hh ligands with high affinity and inhibits Shh-induced changes in gene expression $[18,19]$. Ptch2 is also upregulated in response to Hh signaling, but this can be context dependent [1820]. Additionally, Ptch2 fails to block changes in gene expression induced by a constitutively active form of Smo and is unable to replace Ptch1 function in Ptch1 mutant basal carcinoma cells but does preserve some ligand dependent signaling in Ptch1-null fibroblasts $[19,21,22]$. Interestingly, Ptch1 and 2 can non-autonomously inhibit Smo, possibly through secretion of a cholesterol precursor [10]. Like Ptch1 and Ptch2, Hhip is upregulated in response to Hh signaling. Hhip also binds Hh ligands with high affinity and can attenuate Hh signaling through ligand sequestration $[8,9,23,24]$.
Like Ptch1 and Ptch2, Hhip also negatively regulates the level of Hh ligands to which the responding cell is exposed.

In our studies on Hh signaling in retinal neurogenesis in mice, which begins at $\sim$ E11.5, we've observed that Shh expression can be difficult to detect even though Hh signaling has essential functions in retinal development, and Shh, expressed in retinal ganglion cells (RGCs), is the sole Hh ligand employed during this time (reviewed in [25]). We asked, if Shh expression was lower in the retina than in other anatomical structures, how would Gli1 and the expression of the negative feedback regulators compare? And if differences in expression exist across structures, could anatomical differences correlate with how Shh and the feedback components are expressed? To address these questions, we performed in situ hybridizations for Shh, Gli1, Ptch1, Ptch2, and Hhip on serial sections of an E15.5 embryonic mouse head. Direct comparisons were made for the 5 genes across 6 tissues with active sonic hedgehog signaling.

\section{Main text \\ Materials and methods \\ Animals}

129SvImj mice (stock \#2448, Jackson Laboratory, Bar Harbor, ME) were bred overnight and pregnant dams at gestational day 15.5 were euthanized with a Euthanex EP-1305 $\mathrm{CO}_{2}$ delivery system following AALAC 


\section{(See figure on next page.)}

Fig. 2 Close up comparisons of expression patterns. A, B Schematics of upper (A) and lower (B) molars with buccal side to the left, lingual side to the right. C-G Schematics of stage 3 hair follicle $(\mathbf{C})$, palate $(\mathbf{D})$ eyelid $(\mathbf{E})$, eye $(\mathbf{F})$, and retina $(\mathbf{G})$. H-PP) Expression patterns of Shh (H-N), Gli1 (O- $\mathbf{O})$, Ptch1 (V-BB), Ptch2 (CC-II), and Hhip (JJ-PP) in each structure. See abbreviations list for descriptions. White asterisks indicate histological artifacts where tissues are lacking. The asterisks for the eye structures also indicate the pigmentation of the RPE and is not mRNA staining. Black arrowheads in LL point to Hhip in the condensing mesenchyme surrounding the follicle. White arrows in eyelid panels point to the eyelid signaling field, and the arrowhead denotes a hair follicle. White dashed lines in eye panels denote the iris stroma (below line). White dashed lines in retina panels denote the RPE (below lines) and extraocular mesenchyme including the condensing scleral mesenchyme (above lines)

guidelines. Upon removal from uteri, embryos were rapidly euthanized by decapitation with surgical scissors and heads were placed into Hanks Buffered Saline Solution (HBSS) supplemented with $20 \mathrm{mM}$ HEPES and $6 \mathrm{mg} / \mathrm{ml}$ glucose at room temperature.

\section{In situ hybridization}

Heads were fixed overnight at $4{ }^{\circ} \mathrm{C}$ in $4 \%$ formaldehyde in PBS pH7.5, 2 mM EGTA, followed by cryoprotection with $20 \%$ sucrose/PBS, and frozen in OCT. $12 \mu \mathrm{m}$ serial sections were stained with digoxigenin-labeled anti-sense probes produced by in vitro transcription of sequenceverified linearized plasmids (Additional file 1: Figure S1). Section in situ hybridization was performed as previously described [26-28].

\section{Sample size and data collection}

Five embryos from three separate litters were analyzed. The images shown are from a single animal. Data collection was by visual assessment from two unblinded but independent observers (CLS, EML).

\section{Image capture}

Sections were imaged at $10 \mathrm{X}$ magnification on a Leica DMR microscope under brightfield illumination. Image tiles (8-bit, $1388 \times 1036$ pixel) were acquired with a QICAM Fast 1394 (QImaging, Burnaby, Canada) and automated scanning stage (Märzhäuser, Wetzlar, Germany). Mosaic images were assembled using a Syncroscan montaging system (Synoptics, Frederick, MD). Close up views of the hair follicles were imaged at 20X magnification with a Spot-RT camera (Diagnostic Instruments, Sterling Heights, MI) on a Nikon E-600 microscope using differential interference contrast. Due to their small size, hair follicles could not be analyzed for all probes on adjacent serial sections; similar positions within representative morphologically matched follicles were imaged. Figures were assembled with Photoshop and Illustrator CC (Adobe, San Jose, CA).

\section{Results}

Figure 1 shows the expression patterns of Shh, Gli1 Ptch1, Ptch2, and Hhip in the context of the head. We identified upper and lower molars, palatal rugae, retina, eyelid, and hair follicles as tissues for comparison based on Shh and Gli1 expression. Shh expression identified the cellular sources of Hh signal and was most readily detected in the molars (Fig. 1B). Despite the small sizes of the hair follicles, palatal rugae, and eyelid, Shh expression was still evident at this scale. In contrast, the retina exhibited a low level of Shh expression that was disproportionate to its relatively large size. Gli1 expression, the indicator of Hh signaling, was similarly robust across all 6 tissues (Fig. 1C). Ptch1 expression was also robust in all 6 tissues (Fig. 1D) although its expression in the retina appeared lower by comparison to the levels of Gli1 in each tissue. This is easier observed in Fig. 2. The patterns of Ptch2 were most similar to Shh, although expression levels in the retina and palatal rugae were too low to assess at this scale (Fig. 1E). Hhip was detected in the molars, hair follicle, palatal rugae, and retina; expression in the eyelid was too low to assess. Interestingly, Hhip was abundant in the retina and palatal rugae (Fig. 1F), where Ptch2 was lowest.

Figure 2 shows the expression patterns at scales appropriate for each tissue. Illustrations for each structure are presented (Fig. 2A-G), with specific anatomical and gene expression descriptions provided in the supplement. (Additional file 2). As above, our focus here is to compare gene expression patterns across the structures.

Shh was generally restricted to epithelial tissues within the molars, hair follicles, palatal rugae, and eyelids (Fig. 2H-L). The retina is primarily composed of cells from the neuroepithelium but Shh expression was similarly segregated, in this case, to the differentiated cell layer (DCL) where the RGCs are located (Fig. 2M, N). By and large, Shh expression was robust relative to the size of the tissue except in the retina, where expression was disproportionately lower.

Gli1 and Ptch1 exhibited largely overlapping patterns of expression (Fig. 2O-AA). Both were expressed throughout epithelial and mesenchymal tissues. Interestingly, mesenchymal tissues stained more strongly for Gli1 and Ptch1 in the molars and hair follicles (Fig. 2O-Q, V-X), while epithelial staining was stronger in the palate and eyelids (Fig. 2R, S, Y, Z). In the neural retina, Gli1 expression overlapped with that of Ptch1 in the neuroblast layer 

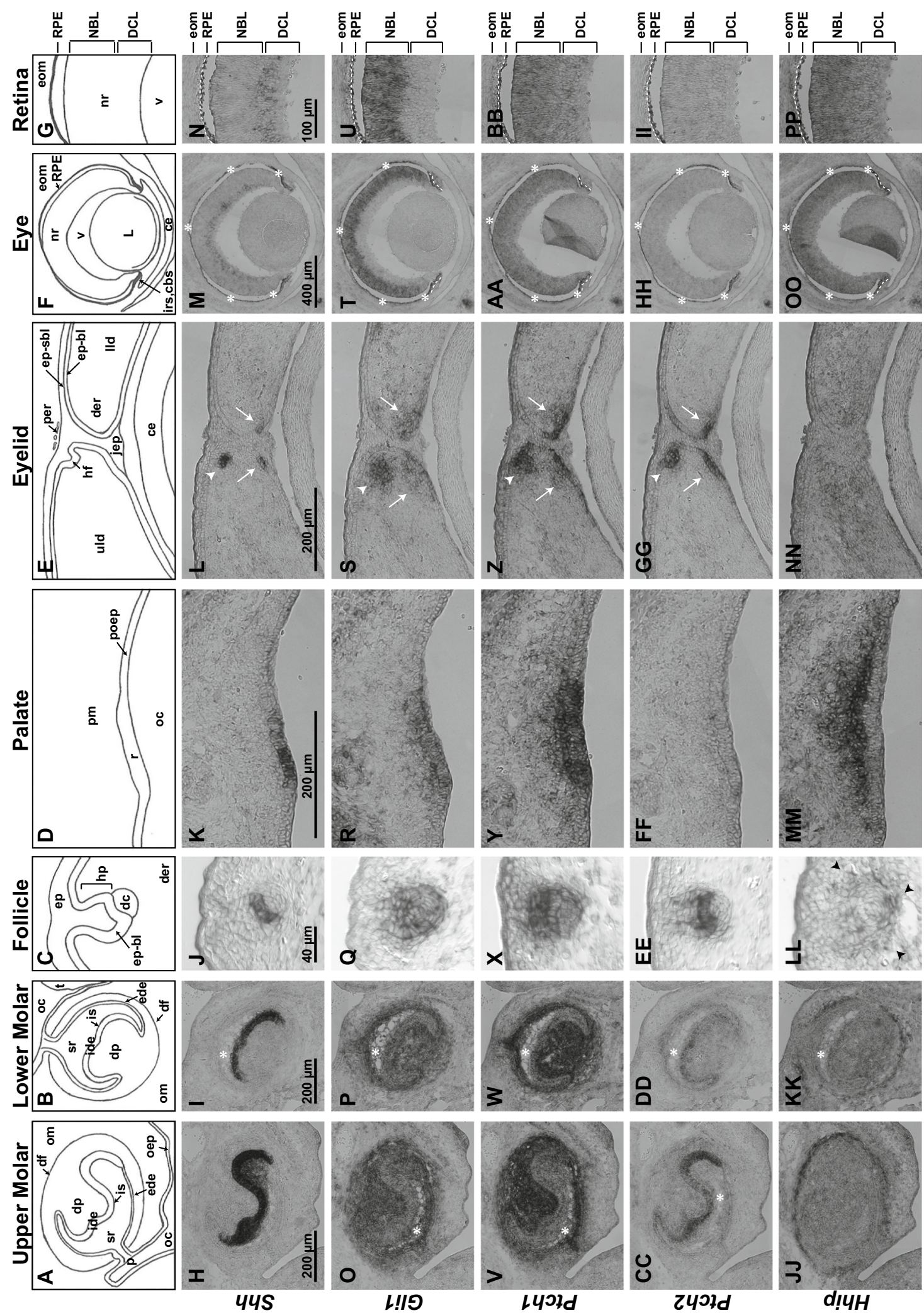

a.
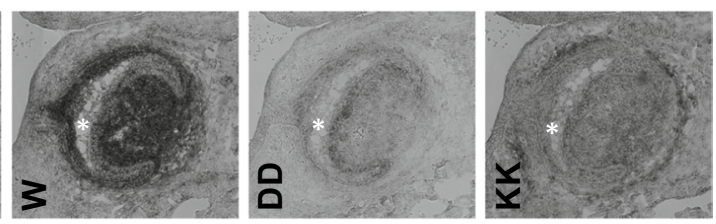

Fig. 2 (See legend on previous page.)
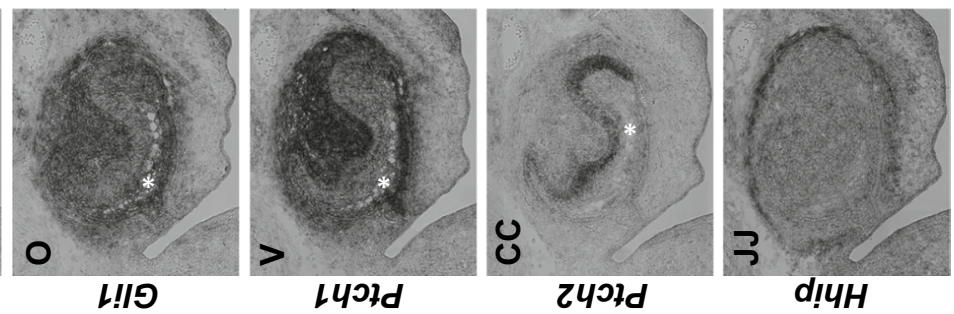
(NBL; Fig. 2T, U, AA, BB). Gli1 was not detected in the DCL whereas Ptch1 extended into the DCL.

Ptch2 overlapped with Shh but was expressed more broadly (Fig. 2CC-EE, GG), consistent with earlier reports $[20,29]$. Two exceptions are the palatal rugae and retina where Ptch2 expression was not detected (Fig. 2FF, HH, II). Since Ptch2 is reliably detected in the retina by transcriptomic and RT-PCR based methods ([28], personal observation (XL and EML), the lack of detection here suggests low and potentially broad expression.

Hhip was expressed in a narrow band in the mesenchyme surrounding the molars at the outer edge of Ptch1 and Gli1 expression, at a distance from Shh-expressing cells (Fig. 2JJ, KK). Although not as distinct as in the molars, Hhip was expressed in the condensing mesenchyme surrounding the epithelial compartment of the hair follicle (Fig. 2LL; arrowheads in Fig. 1F denote additional follicles). Hhip expression within the palate exhibited a graded and robust pattern that was strongest in the palatal mesenchyme (pm) immediately adjacent to the Shh-expressing ruga (r). Expression in the eyelid was too low to assess (Fig. 2NN). As with Ptch1, Hhip expression in the retina extended across both the NBL and DCL in a graded manner that was strongest in the NBL.

\section{Discussion}

Through direct comparative analysis, the expression patterns of several feedback regulators of Hh signaling were assessed. To first address the question that motivated this study, we found that the abundance of Shh mRNA is comparatively low in the retina, but pathway activity, as assessed by Gli1 expression, is robust and on par with other tissues. This suggests tissue-specific differences in how robust signaling is achieved, and selective utilization of the negative feedback factors is one possibility. Supporting this, we observed nonoverlapping expression of Ptch 2 and Hhip, even in structures that express both. Thus, in addition to providing a mechanism to prevent overactive signaling, the utilization of specific feedback inhibitors could contribute to more efficient Hh signaling at lower levels of ligand expression.

Of the three negative regulators, the expression pattern for Ptch1 was most similar to Gli1. Although this makes it the least likely to have a tissue-selective role, it does make it the most reliable of the negative regulators to mark the field of active signaling. This is not surprising since Ptch1 is required in the majority of tissues for ligand-dependent signaling [14]. Subtle differences, however, in its expression levels whether quantitative or spatial, or in the localization or modification of Ptch1 protein, could contribute to tissue-specific influences on signaling [30].

Ptch2 and Hhip, however, exhibited unique expression characteristics. In the molars and hair follicles, their expression domains marked the two ends of the signaling field, with Ptch 2 closest to the source of ligand and Hhip expressed at the outermost extent of signaling. Only Ptch 2 was detected in the eyelid and only Hhip in the palate and retina. Although Ptch2 and Hhip are both negative feedback regulators and act at the level of ligand availability, their differential utilization could account for differences in signaling efficiency across structures. For example, if the retina is most efficient at Hh signaling as suggested, could Hhip have a role in this? How this might occur is not clear but there are differences in how Ptch2 and Hhip regulate ligand availability. Whereas both are on the cell membrane where they bind and remove Shh ligand by endocytosis, Hhip is also secreted and sequesters ligand extracellularly. This could keep ligand intact, releasing it for signaling at a later time or in another location. Thus, Hhip could also have a supportive role in $\mathrm{Hh}$ signaling.

\section{Conclusions}

This study describes the spatial expression patterns of Shh, Gli1, Ptch1, Ptch2 and Hhip in 6 anatomical structures. The patterns of Gli1 and Ptch1 suggest similar levels of signaling across structures with different levels of Shh expression. The patterns of Ptch2 and Hhip suggest different roles in controlling the level of signaling in each tissue.

\section{Limitations}

Colorimetric detection is qualitative and does not allow for precise measurements of mRNA expression levels. Another limitation is that one developmental stage was assessed and temporal differences in gene expression could exist across anatomical structures. However, hedgehog signaling is active before E15.5 in the tissues analyzed so differences due to asynchronous pathway initiation across tissues is unlikely. Another limitation is the indirect nature of using gene expression as indicators of ligand availability or signaling activity. Determining whether differences in Ptch2 and Hhip utilization contribute to qualitatively similar levels of Gli1 expression and pathway activity requires functional perturbations and evaluation of ligand availability for each structure.

\section{Abbreviations}

\section{Molars}

ide: Internal dental epithelium; ede: External dental epithelium; is: Intermediate stratum; sr: Stellate reticulum; dp: Dental papilla; df: Dental follicle; p: Pedicle; oc: Oral cavity; om: Oral mesenchyme; oep: Oral epithelium; t: Tongue. 


\section{Hair follicles}

ep: Epidermis; ep-bl: Basal layer of epidermis; hp: Hair peg; dc: Dermal condensate; der: Dermis.

\section{Palatal rugae}

r: Palatal rugae; poep: Palatal oral epithelium; pm: Palatal mesenchyme; oc: Oral cavity.

\section{Eyelid}

uld: Upper lid; Ild: Lower lid; ep-sbl: Suprabasal layer of epidermis; ep-bl: Basal layer of epidermis; der: Dermis; jep: Junctional epithelium; per: Residual periderm; hf: Hair follicle; ce: Corneal epithelium.

\section{Eye and retina}

nr: Neural retina; RPE: Retinal pigmented epithelium; v: Vitreous; L: Lens; ce: Corneal epithelium; pom: Periocular mesenchyme; irs: Iris stroma; cbs: Ciliary body stroma; NBL: Neuroblast layer; DCL: Differentiated cell layer; eom: Extraocular mesenchyme.

\section{Supplementary Information}

The online version contains supplementary material available at https://doi. org/10.1186/s13104-021-05714-5.

Additional file 1: Figure S1. In situ mRNA hybridization probe templates. cDNA inserts were sequenced from each end, aligned with Blast $N$, and mapped onto their respective NCBI reference sequence using NCBl's Sequence Viewer 3.24.0 (https://www.ncbi.nlm.nih.gov/tools/sviewer/).

Additional file 2: Anatomical descriptions of each structure and gene expression patterns.

\section{Acknowledgements}

We thank Valerie Wallace for the plasmids used for RNA probe synthesis and Robert Marc and Bryan Jones for use of the Syncroscan-equipped Leica DMR upright microscope and imaging assistance.

\section{Authors' contributions}

EML and CLS conceived and wrote the study. CLS performed experiments. $\mathrm{XL}$ sequenced and mapped all probes. EML, CLS, and XL contributed to data analysis, figure preparation, and manuscript editing.

\section{Funding}

This work was supported by funds from the NIH to EML (NEI R01-EY013760), a predoctoral traineeship to CS (NIGMS T32-GM007464), and with unrestricted funds to the John A. Moran Eye Center and the Vanderbilt Eye Institute from Research to Prevent Blindness, Inc.

\section{Availability of data and materials}

The plasmids and datasets used and/or analyzed for the current study are available from the corresponding author on reasonable request.

\section{Declarations}

\section{Ethics approval and consent to participate}

Consent not applicable. Animal use and care were conducted in accordance with protocol 08-1109 approved by the University of Utah Institutional Animal Care and Use Committee and were in compliance with the ARRIVE guidelines.

\section{Consent for publication}

Not applicable.

\section{Competing interests}

The authors have no competing interests to declare.

\section{Author details}

${ }^{1}$ Department of Ophthalmology and Visual Sciences, John A. Moran Eye Center, University of Utah, Salt Lake City, UT, USA. ${ }^{2}$ Department of Ophthalmology and Visual Sciences, Vanderbilt Eye Institute, Vanderbilt University Medical Center, 1161 21st Ave S, B3307 MCN/2569, Nashville, TN 37232, USA. ${ }^{3}$ Department of Cell and Developmental Biology, Vanderbilt University, Nashville, TN, USA.

Received: 3 April 2021 Accepted: 26 July 2021

Published online: 05 August 2021

\section{References}

1. Briscoe J, Therond PP. The mechanisms of Hedgehog signalling and its roles in development and disease. Nat Rev Mol Cell Biol. 2013;14(7):416-29.

2. Varjosalo M, Taipale J. Hedgehog: functions and mechanisms. Genes Dev. 2008;22(18):2454-72.

3. Ramsbottom SA, Pownall ME. Regulation of hedgehog signalling inside and outside the cell. J Dev Biol. 2016;4(3):23.

4. Kong JH, Siebold C, Rohatgi R. Biochemical mechanisms of vertebrate hedgehog signaling. Development. 2019;146(10):dev166892.

5. Lee J, Platt KA, Censullo P, Ruiz i Altaba A. Gli1 is a target of Sonic hedgehog that induces ventral neural tube development. Development. 1997;124(13):2537-52.

6. Regl G, Neill GW, Eichberger T, Kasper M, Ikram MS, Koller J, et al. Human GLI2 and GLI1 are part of a positive feedback mechanism in Basal Cell Carcinoma. Oncogene. 2002;21(36):5529-39.

7. Bai CB, Auerbach W, Lee JS, Stephen D, Joyner AL. Gli2, but not Gli1, is required for initial Shh signaling and ectopic activation of the Shh pathway. Development. 2002;129(20):4753-61.

8. Holtz AM, Peterson KA, Nishi Y, Morin S, Song JY, Charron F, et al. Essential role for ligand-dependent feedback antagonism of vertebrate hedgehog signaling by PTCH1, PTCH2 and HHIP1 during neural patterning. Development. 2013;140(16):3423-34.

9. Kwong L, Bijlsma MF, Roelink H. Shh-mediated degradation of Hhip allows cell autonomous and non-cell autonomous Shh signalling. Nat Commun. 2014:5:4849.

10. Roberts B, Casillas C, Alfaro AC, Jagers C, Roelink H. Patched1 and Patched 2 inhibit Smoothened non-cell autonomously. Elife. 2016:5:e17634.

11. Goodrich LV, Johnson RL, Milenkovic L, McMahon JA, Scott MP. Conservation of the hedgehog/patched signaling pathway from flies to mice: induction of a mouse patched gene by Hedgehog. Genes Dev. 1996;10(3):301-12.

12. Chiang $C$, Litingtung $Y$, Lee $E$, Young KE, Corden JL, Westphal H, et al. Cyclopia and defective axial patterning in mice lacking Sonic hedgehog gene function. Nature. 1996;383(6599):407-13.

13. Marigo V, Tabin CJ. Regulation of patched by sonic hedgehog in the developing neural tube. Proc Natl Acad Sci USA. 1996;93(18):9346-51.

14. Goodrich LV, Milenkovic L, Higgins KM, Scott MP. Altered neural cell fates and medulloblastoma in mouse patched mutants. Science. 1997;277(5329):1109-13.

15. Goodrich LV, Jung D, Higgins KM, Scott MP. Overexpression of ptc1 inhibits induction of Shh target genes and prevents normal patterning in the neural tube. Dev Biol. 1999;211(2):323-34.

16. Butterfield NC, Metzis V, McGlinn E, Bruce SJ, Wainwright BJ, Wicking C. Patched 1 is a crucial determinant of asymmetry and digit number in the vertebrate limb. Development. 2009;136(20):3515-24.

17. Chen Y, Struhl G. Dual roles for patched in sequestering and transducing Hedgehog. Cell. 1996;87(3):553-63.

18. Carpenter D, Stone DM, Brush J, Ryan A, Armanini M, Frantz G, et al. Characterization of two patched receptors for the vertebrate hedgehog protein family. Proc Natl Acad Sci USA. 1998;95(23):13630-4.

19. Rahnama F, Toftgard R, Zaphiropoulos PG. Distinct roles of PTCH2 splice variants in Hedgehog signalling. Biochem J. 2004;378(Pt 2):325-34.

20. Motoyama J, Takabatake T, Takeshima K, Hui C. Ptch2, a second mouse Patched gene is co-expressed with Sonic hedgehog. Nat Genet. 1998;18(2):104-6. 
21. Zaphiropoulos PG, Unden AB, Rahnama F, Hollingsworth RE, Toftgard R. PTCH2, a novel human patched gene, undergoing alternative splicing and up-regulated in basal cell carcinomas. Can Res. 1999;59(4):787-92.

22. Alfaro AC, Roberts B, Kwong L, Bijlsma MF, Roelink H. Ptch2 mediates the Shh response in Ptch1-/- cells. Development. 2014;141(17):3331-9.

23. Chuang PT, McMahon AP. Vertebrate Hedgehog signalling modulated by induction of a Hedgehog-binding protein. Nature. 1999;397(6720):617-21.

24. Chuang PT, Kawcak T, McMahon AP. Feedback control of mammalian Hedgehog signaling by the Hedgehog-binding protein, Hip1, modulates Fgf signaling during branching morphogenesis of the lung. Genes Dev. 2003;17(3):342-7.

25. Wallace VA. Proliferative and cell fate effects of Hedgehog signaling in the vertebrate retina. Brain Res. 2008;1192:61-75.

26. Schaeren-Wiemers N, Gerfin-Moser A. A single protocol to detect transcripts of various types and expression levels in neural tissue and cultured cells: in situ hybridization using digoxigenin-labelled cRNA probes. Histochemistry. 1993;100(6):431-40.
27. Green ES, Stubbs JL, Levine EM. Genetic rescue of cell number in a mouse model of microphthalmia: interactions between $\mathrm{Ch} \times 10$ and $\mathrm{G} 1$-phase cell cycle regulators. Development. 2003;130(3):539-52.

28. Sigulinsky CL, Green ES, Clark AM, Levine EM. Vs×2/Ch×10 ensures the correct timing and magnitude of Hedgehog signaling in the mouse retina. Dev Biol. 2008;317(2):560-75.

29. Motoyama J, Heng H, Crackower MA, Takabatake T, Takeshima K, Tsui LC, et al. Overlapping and non-overlapping Ptch2 expression with Shh during mouse embryogenesis. Mech Dev. 1998;78(1-2):81-4.

30. Cohen M, Kicheva A, Ribeiro A, Blassberg R, Page KM, Barnes CP, et al. Ptch1 and Gli regulate Shh signalling dynamics via multiple mechanisms. Nat Commun. 2015;6:6709.

\section{Publisher's Note}

Springer Nature remains neutral with regard to jurisdictional claims in published maps and institutional affiliations.
Ready to submit your research? Choose BMC and benefit from:

- fast, convenient online submission

- thorough peer review by experienced researchers in your field

- rapid publication on acceptance

- support for research data, including large and complex data types

- gold Open Access which fosters wider collaboration and increased citations

- maximum visibility for your research: over $100 \mathrm{M}$ website views per year

At BMC, research is always in progress.

Learn more biomedcentral.com/submissions 